\title{
Efficient Realization of Classification Using Modified Haar DWT
}

\author{
Rory Mulvaney and Dhananjay S. Phatak
}

\begin{abstract}
The Haar discrete wavelet transform is used as inspiration for a new simple and fast algorithm to train multiclass dyadic decision trees. A localized ordering of the training data changes a multidimensional Haar transform into the one dimensional case, and avoids cache misses. The resulting tree has very simple structure convenient for direct implementation as a fixed-depth threshold network and very fast evaluation of the classification function. The simple structure of the tree is also conducive to good compression of the function. We implement and test the learning algorithm and threshold network but without incorporating sampled interpolated-value points for improved generalization. Compression appears good in low dimensions.
\end{abstract}

\section{INTRODUCTION}

\section{A. Problem Definition}

A seemingly relevant and interesting, yet somewhat neglected problem is how to efficiently approximate with arbitrarily small error a known classification function $\left(\mathbb{R}^{d} \rightarrow \mathcal{S}\right.$, where $\mathcal{S}$ is a set of unrelated symbols) which we can query as an oracle. By "efficiently approximate," we mean having a computationally feasible method of learning a compact yet accurate approximation that we can also evaluate quickly. This basically amounts to storing the entire decision boundary surface in a possibly high dimensional space. To avoid an explosion in spatial complexity, the method needs to have good convergence properties, so that reducing the approximation error by a fraction requires as little additional storage as possible. Because of the problem definition, noise and overfitting are not concerns in the usual sense; instead, we simply desire that there are no large contiguous regions of error on the domain of interest. The oracle to be queried might simply be the k-nearest-neighbor or support vector machine fit of a training set. The usefulness of solving this problem is a potentially great advantage in speed of evaluation if our approximation function is fast compared to the oracle. Speed of evaluation is very important in these days of data mining, as the time-consuming search for correlations between between data might require estimates at large numbers of specified points.

This work was supported in part by NSF grants ECS-9875705 and ECS-0196362.

\section{B. Threshold Network For Speed}

To represent this approximation, it seems a fixeddepth multilayer perceptron (MLP) would offer the ultimate in speed of evaluation. The universal approximation power of single hidden layer MLPs was proven by Hecht-Nielsen in 1989 [8] using the simple observation that any one dimensional function (therefore the sine and cosine functions) may be approximated with a single hidden layer (using sigmoid or threshold transfer functions), and then a multidimensional fourier transform can be applied to extend this capability to multidimensional functions. Of course in practice, constructing a neural network for classification functions this way would require many hidden nodes, because many modes of the multidimensional fourier series may be required, and each of these modes (a sine or cosine) requires many hidden nodes (with sigmoid or threshold transfer functions) to approximate accurately.

Because of this and the fact that class values are fundamentally different from the real values approximated by a fourier transform, we turned instead to a multidimensional Haar discrete wavelet transform (DWT) [18], since it is piecewise constant and therefore much easier to represent class values using threshold nodes. (We make several modifications to the Haar DWT, including one that allows it to work with multiple class values, rather than real values.) However, whereas the multidimensional fourier transform consists of a sum of one dimensional fourier components (the 1-D components lie parallel to different vectors in the multidimensional frequency space), the wavelet transform consists of a sum of multidimensional Haar wavelet components. The net effect of all this is that 2 hidden layers are required to represent the DWT, where the output of each node in the second hidden layer basically represents the value of a Haar component. So our approximation of the function will be represented by a 2 hidden-layer threshold network.

We further show how everything after the first hidden layer of this network (first layer is required, since the inputs are not necessarily boolean) can be efficiently implemented with three levels of boolean logic (ignor- 
ing inverters). According to [6], [15], the jump from two to three levels of boolean logic can dramatically reduce the number of gates required, while little improvement is had by increasing the number of layers further. Therefore, it would be very hard to come by a faster-evaluating architecture with which to represent functions (without a large increase in gates).

In addition to the interpretation as a fixed-depth MLP or boolean logic network architecture, this Haar DWT essentially learns a forced-split "dyadic decision tree," as introduced recently in [16]. Dyadic decision trees are binary decision trees with decision nodes that always bisect their domain along a predetermined hyperplane parallel to a coordinate axis. The decision planes down any path in the tree simply cycle through the $d$ coordinates in a forced manner. These are called forced splits; it is also possible to have free splits, where the optimal coordinate to split on can be independently chosen at any given node. In [17] a method was sketched (not very explicitly) for how to construct 2 hidden-layer MLPs (very much like the ones we construct in this paper) from an existing decision tree. They then trained the resulting MLP using gradient descent for further slight improvement in generalization. We don't deal with decision trees here, but one can actually perform the multiclass Haar DWT directly on the nodes of a binary decision tree to help analyze and rebalance the tree [11]; assuming the decsision nodes all represent linear boundaries, these wavelet coefficients each represent actual convex polytopic regions of space, making them more meaningful (easier to interpret) than decision nodes.

Due to their good compression and function approximation characteristics, wavelets have been used in the past to construct neural networks [1],[19], but implementing a multidimensional wavelet transform for dimensions above 2 or 3 has been seen as difficult [10] and seems to be generally avoided. Though there are many possible multidimensional Haar DWT decompositions, we have found that sorting the data into a certain order reduces the problem of performing a certain type of multidimensional Haar DWT into the trivial case of performing a one dimensional DWT on the reordered data. Thus the DWT itself is very easy to implement and fast to execute.

In this paper we simply test the generalization error of the wavelet transform on two benchmarks and note the size of the resulting threshold network. Generalization error is somewhat sub-par, but it could be improved dramatically through techniques like bagging [7] or targeted sampling of the k-nearest neighbor solution.

\section{Multiclass Modified HaAR DWT}

We begin with a small example to demonstrate the simple operation of a one-dimensional Haar DWT. Due to space limitations, the reader can refer to [4], [18] for a more rigorous explanation of wavelets. In Figure 1] the top row is the data being transformed, and the resulting wavelet coefficients are in the bottom rows of

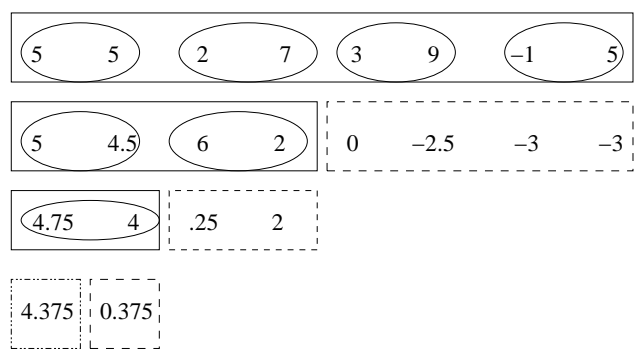

Fig. 1

HAAR DWT EXAMPLE

each column. Actually, the coefficient in the far lowerleft box is not a "wavelet" coefficient, but rather the residual "scaling" coefficient. From this figure, it is very easy to understand the Haar wavelet transform. Starting at the top row, elements are paired off (indicated by the circles). The four average values of theses four pairs are placed in the second row in the left box. The "halved difference" of these four pairs are placed in the right box in the second row. These are the finest scale wavelet coefficients. The values in the left box represent an approximation of the original data (from a "low pass" filter), while values in the right box are the "details" from a "high (frequency) pass" filter. It is easy to see how to reconstruct the original data from these means and differences. This procedure is performed recursively on the left half (the low-pass coefficients) until the data has been reduced to the desired number of low-pass coefficients, in this case, the minimum, 1.

There are actually many different ways of performing a multidimensional wavelet transform. In a primer on wavelets [18], two methods for performing multidimensional Haar DWTs are discussed. Regarding the actual shape and form of the multidimensional wavelets, we basically use the second method, which they refer to as the "nonstandard method." The operation of this transform is simple. Figure 2 shows the two dimensional analog of low pass coefficient pairings (the circled pairs) to compute the "means and differences" in four consecutive levels of the algorithm. At each level, the algorithm simply alternates between pairing neighbors 


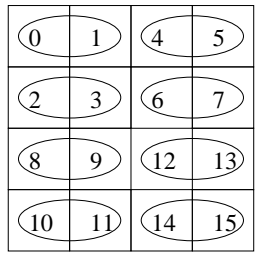

(a)

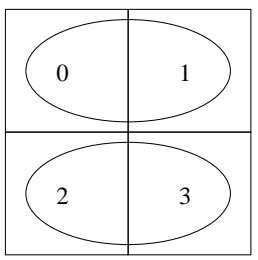

(c)

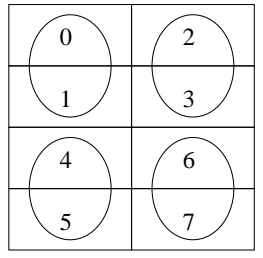

(b)

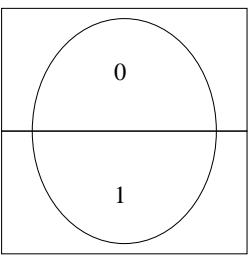

(d)
Fig. 2

MulTidimensional GROUPING PROGRESSION

in the horizontal direction and pairing on the vertical direction.

In this progression of figures, we've labeled each cell with a number indicating the serialized index of each two-dimensional data point. Notice that this is not the usual row-major ordering of data that is typically used in a d-dimensional matrix. Also notice that every pairing joins cells with consecutive indices (also as it was in the one-dimensional transform in Figure 1]. Thus, this 2-D transform can be more easily performed as a 1-D transform on the data sorted in this manner.

It turns out that this ordering of the data generalizes to any dimensionality, so that any d-dimensional Haar transform may be performed as a one-dimensional Haar DWT. This ordering can be simply described as the ordering induced by interleaving the bits of a point's d-dimensional coordinates into a single coordinate. For example, if $x_{1}$ represents the most significant bit of the $\mathrm{x}$-coordinate, and $x_{n}$ represents the least significant bit, $x_{1} y_{1} z_{1} x_{2} y_{2} z_{2} \ldots x_{n} y_{n} z_{n}$ is the merged, interleaved index for a three dimensional point.

To visualize this ordering of the data in two dimensions, refer to figure 3 The numbers in the figure represent the serialized indices of the elements in the corresponding blocks of the matrix. It has a natural block-structured recursive hierarchy that corresponds nicely with the bit pattern of the serialized index:

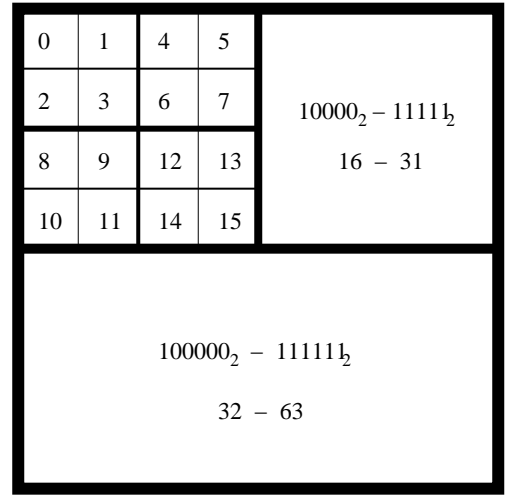

Fig. 3

SERIALIZATION OF DATA In A TWO-DimensionAl Matrix

\begin{tabular}{|l|l|l|l|l|l|l|l|l|l|l|l|l|l|l|l|}
\hline $\mathrm{b}$ & - & - & $\mathrm{g}$ & $\mathrm{c}$ & - & $\mathrm{g}$ & $\mathrm{b}$ & $\mathrm{r}$ & $\mathrm{r}$ & $\mathrm{y}$ & - & $\mathrm{p}$ & - & - & $\mathrm{g}$ \\
\hline
\end{tabular}

\begin{tabular}{|l|l|l|l|l|l|l|l|l|c|c|c|c|c|c|c|}
\hline $\mathrm{b}$ & $\mathrm{g}$ & $\mathrm{c}$ & $\mathrm{b}, \mathrm{g}$ & $\mathrm{r}$ & $\mathrm{y}$ & $\mathrm{p}$ & $\mathrm{g}$ & $\begin{array}{ccc}0 \\
0\end{array}$ & 0 & 0 & $?$ & 0 & 0 & 0 & 0 \\
\hline
\end{tabular}

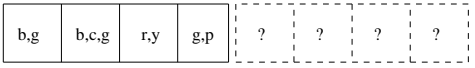

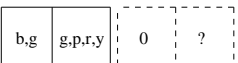

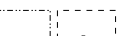

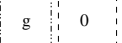

(a)
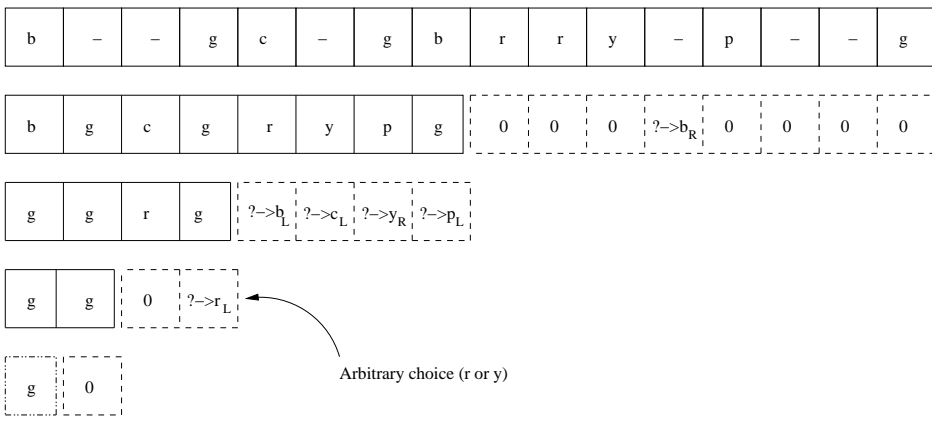

(b)

Fig. 4

Multiclass HaAR DWT EXAMPLE. 
the bits of the serialized index tell which path to take through a binary tree whose leaves are the data elements of the matrix. In the 2-D example of the figure, the most significant bit of the index tells whether the data lies in the upper or lower half of the matrix, the second bit tells whether it lies in the right or left half of that half, and so on.

Now that the multidimensional Haar DWT has been completely specified, we describe our modifications to handle multiple class values and "unknown" regions. Figure 4 is intended as an instructive, perhaps unrealistic, example of the algorithm. This is similar to the previous example from Figure 1 except that we now have class values represented by letters (standing for the colors black, cyan, green, purple, red, and yellow) instead of real numbers, and there are also unknown regions of the function, represented by dashes. For instructive purposes we explain the algorithm in two phases. The first phase, illustrated in Figure 4(a) involves establishing "candidate sets" of low-pass coefficients. Instead of taking the mean of two low-pass coefficients to produce the low-pass coefficient at the next level, we take the intersection set of the two candidate sets, if there is an intersection. If there is no intersection, we take the union set. The dashes representing unknown regions are treated as "don't cares," so they effectively take the same value as the neighboring region. Whenever there is an intersection between the candidate sets, we also set the corresponding high-pass coefficient (recall this was the "halved-difference" in the standard Haar DWT) to zero. A high-pass coefficient corresponding to a candidate set formed as the union of two candidate sets is determined later, and represented as a '?' in the first phase figure.

In the second phase, completed in Figure 4(b) all candidate sets are narrowed to a single class value and all undetermined high-pass coefficients are also determined. Observe that the low-pass coefficients form a binary tree hierarchy (from how they were calculated) rooted at the final low-pass coefficient. Whenever there is a singleton candidate set, the candidate sets of all descendents of that node in the tree can be determined by the algorithm. If the tree is rooted by a non-singleton candidate set, the algorithm narrows the set to a single random candidate class. Once the set of a parent node has been narrowed, its two children are narrowed to the same class as the parent. It's possible that one of the children's sets doesn't contain the class of the parent. In this case, that child's class can be assigned randomly from its candidate set, and the appropriate high-pass coefficient at the next level (indicated by the arrow in
Figure 4(b) is assigned to indicate the right or left child has a different value from the parent (' $L$ ' and ' $R$ ' subscripts of the high-pass coefficients in the figure indicate whether the class applies to the left or right child). This process is repeated recursively until all coefficients in a given subtree have been uniquely determined. In [11] we show that this algorithm effectively uses dynamic programming and uses the minimum number of non-zero wavelet coefficients within the framework to capture the function at the training points (obviously there is never any training set error).

Note that neither the time or space complexity of the algorithm is affected by the number of don't-cares, since they don't actually need to be stored or ever even acknowledged by the algorithm. When the algorithm pairs off neighboring points in the serialized array, it simply needs to check their coordinates to verify that the points really are neighbors at the given resolution; otherwise, there is an implicit unknown point between them. Thus, given $n$ original data points, only $2(n-1)$ coefficients are computed, so after the data has been sorted, the run time is linear in $n$. More generally, it is bounded by $O(n b k)$, where $k$ is the number of classes and $b$ is the total number of bits required for the coordinates of each point. One should also note that all memory accesses are highly localized, likely in stark contrast to other multidimensional algorithms. Thus this algorithm should encounter a minimal number of memory cycle delays, making it extremely fast.

\section{CONVERSiOn to FAUlt-TOLERANT THRESHOLD NETWORK}

A useful intuitive image is that the non-zero high-pass coefficients represent colored hyper-rectangular stickers which are placed on top of one another (in overruling fashion) in regions within the dyadic framework (such as those regions in Figure 2). The largest sticker corresponds to the last low-pass coefficient, and it shows through in areas where it isn't overruled by smaller stickers (higher-resolution coefficients) that "cover" it.

Now Figure 5] illustrates the architecture of the threshold network. Each node in the first hidden layer implements a hyperplane parallel to a coordinate axis, so they each require only one of the $d$ inputs and a bias. A hashtable is used to ensure identical nodes aren't created. Each node in the second hidden layer corresponds to a non-zero high-pass coefficient, or hyper-rectangular sticker. These nodes are activated iff the input to the network is inside the region of the corresponding sticker; thus it is activated iff the input is inside all $2 d$ hyperplanes that delimit the hyperrectangular region. 

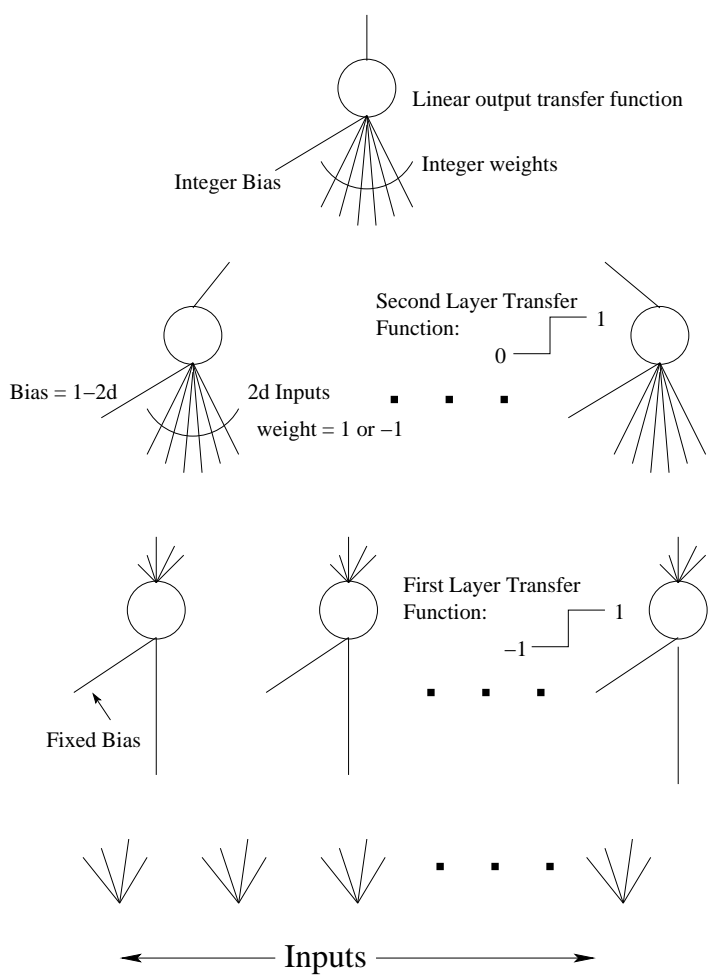

Fig. 5

ARCHITECTURE OF THE THRESHOLD NETWORK.

Output nodes output the weighted sum of their inputs and bias, so each class needs to have a numerical assignment. To understand how to set the weights to the output units, notice a general point $p$ is inside the sticker-regions corresponding to some "signature" set $\mathcal{S}=\left\{r_{0}, r_{1}, r_{2}, \ldots, r_{k}\right\}$ of wavelet coefficients. This set of regions always has the property that region $r_{i}$ completely contains region $r_{i+1}$. Also note that all points whose "signature" set is equal to $\mathcal{S}$ are classified identically as $p$ by the wavelet-transformed function. Let $w_{i}$ represent the weight of the output connection from the node representing region $r_{i}$ ( $w_{0}$ represents the bias due to the final low-pass coefficient). Then given input point $p$, the output of the network is clearly $\sum_{i=0}^{k} w_{i}$. Regardless of the value of the sum $\sum_{i=0}^{k-1} w_{i}$, we can always set $w_{k}$ so that the network outputs the correct value for point $p$ (and all points with $p$ 's signature set). These weights can be computed efficiently in a depth-first traversal of the tree structure of the wavelet coefficients.

To use simpler boolean output units, it is straightforward to simply change the output layer and connections to have $\left\lceil\log _{2} k\right\rceil$ boolean outputs, for $k$ classes. All weights to the output layer would now be 1 or -1 . However, now the amount of fan-in to the output nodes becomes slightly sensitive to the assignment of classes to integers. Since it's a dense coding of classes, it would seem difficult to reduce fan-in very much without trial and error, but perhaps assigning the classes with the most non-zero coefficients to the integers with the fewest bits on would help.

The original motivation of this work was to construct a fault tolerant MLP. Since all weights to the boolean output layer are 1 or -1 , making the network completely fault tolerant to single faults is as simple as making two replications (for triple modular redundancy) of each subnet that feeds the output layer, as described in [13]. There it was proven the minimum number of replications required for fault tolerance is 2 . Previous work [12], [14] was unable to achieve this lower bound.

\section{Conversion to Boolean Network}

Finally, we describe the simple conversion of everything after the first hidden layer of the threshold network (the inputs aren't necessarily boolean) to a AND-AND-OR boolean logic circuit of about the same size. The first layer consists of an AND gate for each non-zero wavelet coefficient. Each outputs 1 whenever the input is inside the hyper-rectangular sticker-region for its corresponding coefficient. Thinking in 2-D, there is a colored sticker at the bottom that shows through in many places. Those stickers directly on top of the bottom sticker are its "exceptions." We use a $2^{\text {nd }}$ level AND gate to represent this by ANDing the bottom sticker with the NOT of each of the stickers directly on top of it. Now recursively treat the "exceptions to the exceptions" as if they were the bottom sticker, making $2^{\text {nd }}$ level AND gates for each of them. All $2^{\text {nd }}$ level AND gates, as well as some $1^{\text {st }}$ level AND gates who don't have exceptions, are input to the output OR gate.

\section{EXPERIMENTS}

\section{A. Benchmark Results}

We applied the wavelet transform directly to the training sets of two benchmarks without any extra interpolated sampling or bagging, constructed a threshold network, and measured generalization error. The vowel benchmark [2] deals with 11-class data in a 10dimensional space. Respective train and test sets have 528 and 462 patterns. The resulting threshold network had 73 and 132 nodes in the first and second hidden layers, and had $60.6 \%$ generalization error on the test set (this is a difficult benchmark). For comparison, [7] tabulates the performance of 17 other methods. Only 
3 of of them beat nearest neighbor, which had $44 \%$ error (the best performer had 39\%). The CART decision tree [7] had $56 \%$ error, and a linear perceptron tied for worst, at $67 \%$ error.

For the popular two spirals benchmark we generated 386 patterns using the code from [5]. We split this into training and test sets of 194 and 192 by taking every other pattern from each spiral. The threshold network had 36 and 57 hidden nodes in the first and second layer, and had $5.7 \%$ generalization error. Each of the 58 wavelet coefficients is a single bit and an address. Storing them in a tree format would probably require about 30 bytes or less.

\section{B. Pen \& Paper Results}

Arguably, simple examples are more indicative of performance. Since decision boundaries of most practical functions are locally linear, we examined, for different linear boundaries, whether we can achieve convergence such that the error goes like $O\left(\frac{1}{n}\right)$, where $n$ is the number of wavelet coefficients (size of the approximation). This appears possible in 2 and 3 dimensions, though boundaries offset from "center" seem to require considerably more coefficients than a dyadic decision tree with "free splits."

In higher dimensions, convergence looks decidedly bad for linear function boundaries normal to the vector $(1,1,1, \ldots, 1)$. Error can successfully "hide in the corners" in high dimensions, apparently due to inflexibility caused by requiring the decision planes be parallel to a coordinate axis. For a decision tree architecture, this suggests allowing low precision hyperplane bisections normal to vectors whose entries are in $\{-1,0,1\}$.

\section{Vi. Conclusions, Speculation, and Future WORK}

The multiclass Haar DWT is very efficient, elegant, and simple. The coefficients have a very useful interpretation as regions of space, allowing us to easily construct fairly small, very fast-evaluating fixed depth boolean or threshold networks, and even help to balance general binary decision trees. However, despite the good reputation of wavelets for compression, it seems performance may fade quickly with increasing dimension. Better dimensionality reduction always pays off.

We think a decision tree architecture with low precision linear combination splits might help asymptotic convergence rates, though this approach has been criticized [3]. Recent advances in uniform sampling [9] may have made this strategy ultimately feasible. The resulting decision tree can still be realized as a fixed depth boolean or threshold network.

\section{REFERENCES}

[1] Bhavik Bakshi, Alexandros Koulouris, and George Stephanopoulos, Wave-nets: Novel learning techniques, and the induction of physiceally interpretable models, SPIE Vol. 2242 Wavelet Applications, 1994, pp. 637-648.

[2] C.L. Blake and C.J. Merz, UCI repository of machine learning databases, http://www.ics.uci.edu/ mlearn/MLRepository.html, 1998.

[3] Leo Breiman and Jerome H. Friedman, Tree-structured classification via generalized discriminant analysis: Comment, Journal of the American Statistical Society (1988), no. 403, 725-727.

[4] Adhemar Bultheel, Learning to swim in a sea of wavelets, Bulletin of the Belgian Mathematical Society - Simon Stevin 2 (1995), 1-45.

[5] $C M U$ repository neural benchmarks, http://www-2.cs.cmu.edu/afs/cs/project/airepository/ai/areas/neural/bench/cmu/bench.tgz.

[6] Debatosh Debnath and Tsutomu Sasao, A heuristic algorithm to design AND-OR-EXOR three-level networks, Asia and South Pacific Design Automation Conference, 1998, pp. 69-74.

[7] Trevor Hastie, Robert Tibshirani, and Jerome Friedman, The elements of statistical learning - data mining, inference, and prediction, Springer, 2001.

[8] Robert Hecht-Nielsen, Theory of the backpropagation neural network, Proceedings of International Joint Conference on Neural Networks (Washington, DC), vol. 1, 1989, pp. 593-605.

[9] Ravi Kannan, Laszlo Lovasz, and Miklos Simonovits, Random walks and an $O^{*}\left(n^{5}\right)$ volume algorithm for convex bodies, Random Structures and Algorithms 11 (1997), no. 1, 1-50.

[10] Tharmarajah Kugarajah and Qinghua Zhang, Multidimensional wavelet frames, IEEE Transactions on Neural Networks 6 (1995), 1552-1556.

[11] Rory Mulvaney and Dhananjay S. Phatak, Efficient realization of classification in sparsely featured high dimensional input spaces using modified haar DWT, Tech. Report TR-CS-03-21, University of Maryland, Baltimore County, January 2003.

[12] Dhananjay S. Phatak, Fault Tolerant Artificial Neural Networks, Proceedings of the 5th IEEE Dual Use Technologies and Applications Conference (Utica/Rome), May 1995, pp. 193 - 198.

[13] Dhananjay S. Phatak and I. Koren, Complete and partial fault tolerance of feedforward neural nets, IEEE Transactions on Neural Networks 6 (1995), no. 2, 446-456.

[14] Dhananjay S. Phatak and Elko Tchernev, Synthesis of fault tolerant neural networks, Proceedings of International Joint Conference on Neural Networks (Honolulu, Hawaii), May 2002.

[15] Tsutomu Sasao, OR-AND-OR three-level networks, Representations of discrete functions (Tsutomu Sasao and Masahiro Fujita, eds.), Kluwer Academic Publishers, 1996.

[16] Clayton Scott and Robert Nowak, Dyadic classification trees via structural risk minimization, Proceedings of Conference on Neural Information Processing Systems (Vancouver, Canada), December 2002.

[17] Ishwar K. Sethi, Decision tree performance enhancement using an artificial neural network implementation, Artificial Neural Networks and Statistical Pattern Recognition: Old and New Connections (Ishwar K. Sethi and Anil K. Jain, eds.), Machine Intelligence and Pattern Recognition, vol. 11, North-Holland, 1991, pp. $71-88$.

[18] Eric J. Stollnitz, Tony D. DeRose, and David H. Salesin, Wavelets for computer graphics: A primer, part 1, IEEE Computer Graphics and Applications 15 (1995), no. 3, 76-84.

[19] Qinghua Zhang and Albert Benveniste, Wavelet networks, IEEE Transactions on Neural Networks 3 (1992), 889-898. 